Paediatrica Indonesiana $14: 113$ - 11\%.July - August 19\%4. 113

From the Gastroenterological Research Unit, Princess Margaret

Children's Medical Research Foundation, Perth, Western Australia.

\title{
The Use of Medium-Chain Triglyceride in Children with Intestinal Malabsorption
}

by

MICHAEL GRACEY ${ }^{1,2}$

Medium-chain triglycerides (MCT) are fats in which the chain length is six to twelve carbon atoms. This is in contrast to the more usual dietary sources of fats, long-chain triglycerides (LCT), which contain more than 14 carbon atoms in their atomic chain. Most naturally occurring triglycerides are LCT and are found in common dietary constituents such as animal fats and dairy products. However coconut oil contains more than $10 \% \mathrm{MCT}$ and is used as the raw material for the commercial production of MCT oils which are obtained by fractionation..

The differences in chemical structure between LCT and MCT are important because they are related to major differences in the characteristics of absorption and metabolism of these two classes of lipids (Figure 1).

Unlike LCT, significant absorption of MCT occurs in the absence of bile salts and pancreatic lipase. Intestinal uptake and transport of MCT are rapid, even when very little intraluminal lipase is present (Isselbacher, 1966). MCT cross the epithelial cells of the gut rapidly, the later stages of absorption are different from LCT as most of the MCT travel directly to the portal blood stream without going through the steps of intracellular re-esterification and formation of chylomicrons (Bloom et al., 1951). After reaching the liver, MCT are rapidly oxidised to 2-carbon fragmenits which are available for different metabolic processes (Scheig, 1968).

These physiologial differences between LCT and MCT have led to the use of MCT in various disorders where malabsorption of LCT occurs but where MCT can be absorbed because they bypass several of the

1. Adolph Basser Research Fellow of the Royal Australasian College of Physicians.

2. Supported by a Grant from the Welcome Trust, London. 
steps necessary for the absorption of long chain fats. These include pancreatic disease, chronic liver disease, disorders of the biliary tract and diseases of the small intesitinal epithelium and lymphatic vessels. Over recent years commercial preparations of MCT have become wildly available, particularly in Europe, Nor'h America and Australia, and the usefulness of these preparations in various clinical situations has been well established (Holt, 1967; Senior, 1969). The place of MCT in paediatrics has also been reviewed recently (Gracey, Burke and Anderson, 1967). The commenits which follow are a brief summary of the therapeutic place of MCT in children with malabsorption. This will be followed by some remarks about the potential value of MCT in countries like Indonesia where the commonest cause of chronic diarrhoea and malabsorption in children is malnutrition and chronic and repeated gastrointestinal infections and infestations and where MCT has not been used widely.

\section{Pancreatic disease}

In Westernized countries, such as Australia, the commonest cause of exocrine pancreatic insufficiency in children is cystic fibrosis of the pancreas in which there is usually severe steatorrhoea due to lack of pancreatic enzyme secretion. MCT are a useful additional source of calories for these children but do not appear to improve long-term weight gain or eventual prognosis. In developing or pre-industrialized countries, mainutrition is probably the commonest cause of exocrine pancreatic insufficiency. As can be seen from Figure 2, an MCT diet leads to improvement of faecal fat excretion in children with pancreatic insufficiency and on theoretical grounds could be useful in various forms of malabsorption due to pancreatic disease, whatever the underlying cause.

\section{Liver disease}

Burke and Danks (1966) showed that steatorrhoea is common in infants and children with liver and biliary disease such as neonatal hepatitis, biliary atresia and cirrhosis and thait MCT are effective in reducing the amount of fat lost in the stools in these patients (Figure 3).

\section{Small bowel diseases}

MCT are able to be absorbed rapi dily by the small intestine despite the presence of diffuse mucosal disease and loss of surface area, as in coeliac disease. In some severely ill partients MCT may help induce weight gain whilst awaiting response to a gluten-free diet which is the specific treatment for this disorder. It can also be used as a source of calories in infants and children with intestinal malabsorption of uncertain aetiology where the main aim is to improve weight gain and the patient's clinical state before complete investigation can be undertaken safely. 


\section{ABSORPTION OF LONG AND MEDIUM CHAIN FATS}
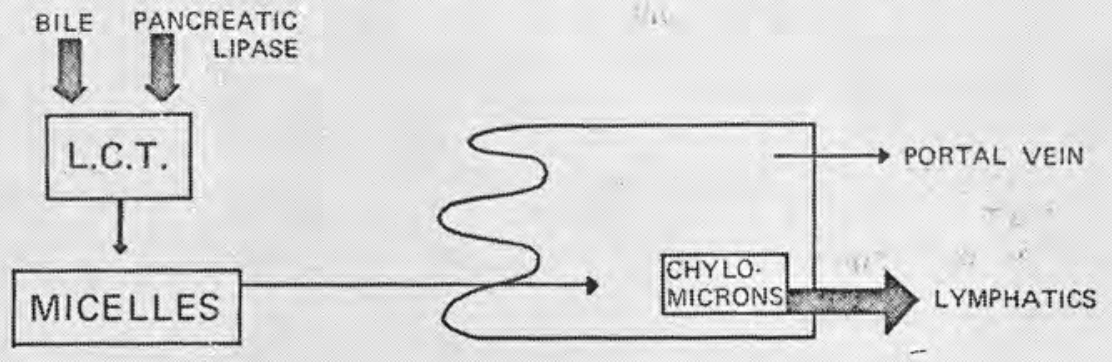

LUMEN

CELL

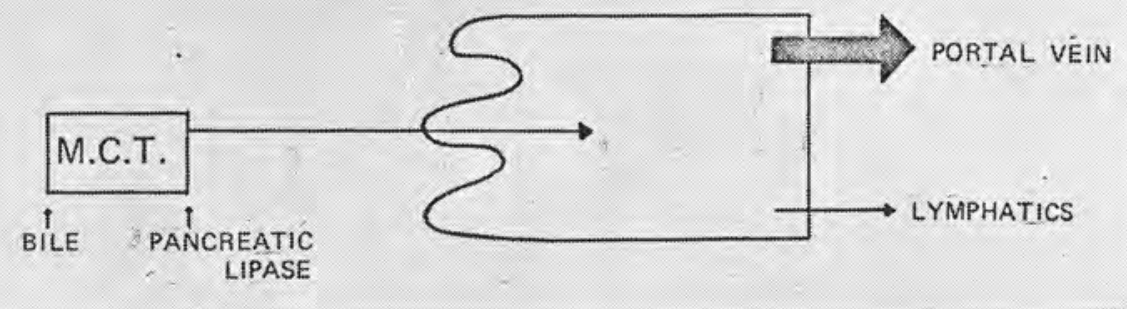

FIG. 1 : Differences between absorption of long-chain triglycerides (LCT) and medium-chain trigiycerides (MCT) as explained in the text. Reproduced from the Australian Paediatric Jounnal with kind permission of the Editor and the authors, Burke and Anderson (1967). 


\section{CYSTIC FIBROSIS}

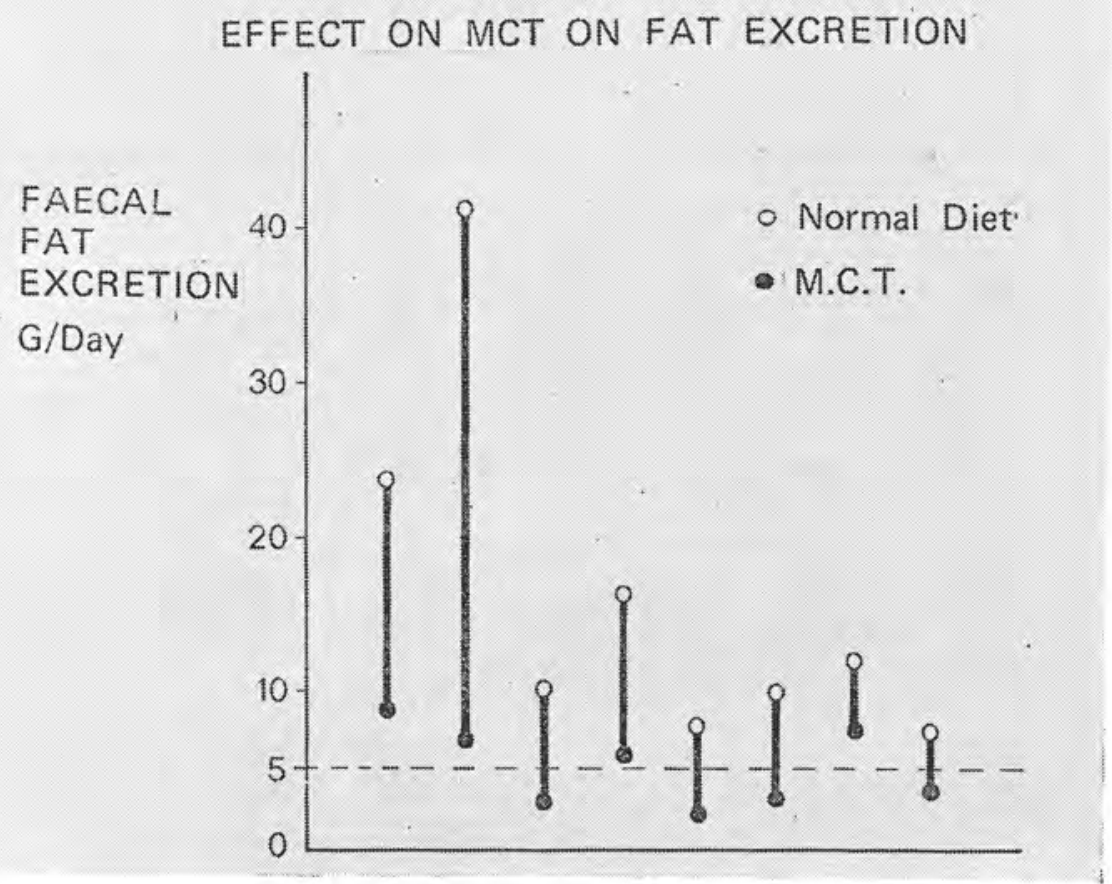

FIG. 2 : Decrease in stooi fat excretion in children with cystic fibrosis of the pancreas when an an MCT-containing diet. Reproduced with kind permission of the Editor of the Australian $\mathrm{Pa}$ ediatric Journal and the authors, Burke and Anderson, 196\%. 


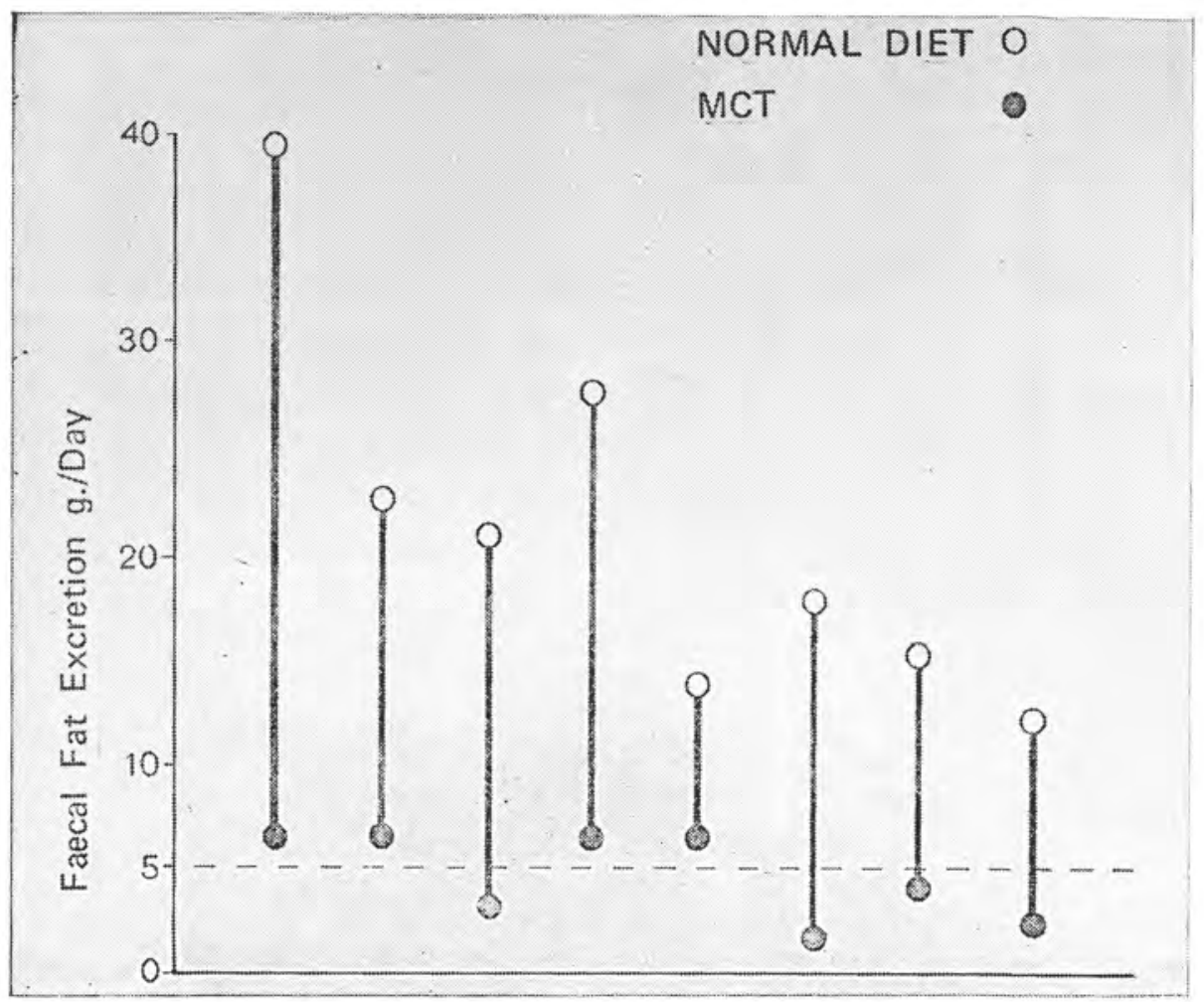

FIG. 3 : Reduction of stool fat excretion in four patients with biliary atresia (left) and four patients with neonatal hepatitis when put on an MCT-contaning diet. Reproducea with kina permission of the Editor the Austraiian Paediatric and the authors, Burke and Anderson, 1967. 
MCT have also been useful in the dietary management of young patients following extensive intestinal resection which can lead to malnutrition, impaired growth and deficiency states (Burke and Anderson, 1967). When using MCT preparations in patients following gastrointestinal surgery, particular attention must be paid to the sugar content of the formula as these patien's may have sugar initolerance which will cause severe diarrhoea if it is not recognized and treated by removal of the affected sugar from the patienit's diet-in most cases lactose will be the sugar not tolerated and a lactose-free, MCT-containing formula will need to be used. Such preparations are available commercially.

\section{Lymphatic diseases}

As mentioned above, MCT are not transported through the intestinal lymphatics but itravel to the portal blood stream. An MCT diet may, therefore, be useful in Iymphatic disorders such as congenital lymphangiectasia or acquired (e.g. trauma or tuberculosis) of the intraabdominal lymphatic vessels. (Tamir et al., 1968; Gracey and Gooch, 1968). In such situations MCT will be absorbed into the portal blood and transported to the liver whereas LCT would either leak out of the damaged lymphatic vessel or would not be absorbed because of blockage of the lymphatic drainage system.

\section{MCT in children with malnutrition}

Diarrhoea is a characteristic feature of malnourished children; its pathogenesis is complex and multifactorial.

Included amongst these factors are:

(i) histological abnormalities of the small intestinal mucosa,

(ii) secondary disaccharidase deficiency caused by the histological abnormalities,

(iii) impaired absorption of nutrients,

(iv) repeated infections and infestations in the gut,

(v) exocrine pancreatic insufficiency,

(vi) liver dysfunction, and

(vii) microbial contamination of the upper gut.

In a recent study (Gracey et al., in press) steatorrhoea was found commonly in young Indonesian children with protein-calorie malnutrition, using the short term lipiodol absorption test as a screening procedure for malabsorption of fat. Although it is not possible to say which factors are most important in causing malabsorption in these children, it is reasonable to suspect that a combination of small bowel disease and impaiment of hepatic and pancreatic function are involved. On theoretical grounds, at could be anticipated that MCT would be absorbed much better than LCT in such children and that MCT would be a useful source of dietary calories in the early 
stages of nutrittional rehabilitation in these patients. It must be remembered, of course, that these children have a high incidence of sugar intolerance as well as malabsorption of fat and this must be taken into account when planning their dietary management; because lactase deficiency is so common in these children feeding formulae containing a significant quantity of lactose ought to be avoided. Recently, a lactose-free, MCT-containing preparation has been used with success in malnourished children in South America (Graham et al., 1973). Sutedjo (1974) has very recently advocated the use of MCT in malnourished children in Indonesia. Admittedly, the limiting factor about this suggestion is the high cost of MCT preparations and their relative unavaillability. Despite this, it seems desirable that carefully planned clinical trials should be conducted to test the therapeutic value of MCT in this part of the world. Furthermore, as pointed out by $\mathrm{Su}$ tejo (1974) attempts should be made to provide predominantly MCT-containing formulae from vegetable oils, such as coconut oil, which are readilly available in Indonesia and should, therefore, be much less expensive. It is hoped that the technical expertise of the pharmaceutical industry will accept this important challenge to their inventiveness.

\section{REFERENCES}

1. BLOOM, B., CHAIKOFF, I.L., and REINHARDT, W.O.: Intestinal lymph as pathway for transport of absorbed fatty acids of different chain lengths. Amer. J. Physiol. 166 : 451 (1951).

2. BURKE, V., and DANKS, D.: Medium chain triglyceride diet. Its use in treatment of liver disease. Brit. med. J. 2: 1050 (1966).

3. BURKE, V., and ANDERSON, C.M. : Experience with medium chain triglycerides in malabsorptive states in childhood. Aust. paediat. J. 2 : 114 (1967).

4. GRACEY, M., BURKE, V., and ANDERSON, C.M.: Assessment of medium chain triglyceride feeding in infants with cystic fibrosis. Arch. Dis. Childh. 44 : 401 (1969).
5. GRACEY, M., BURKE, V., and ANDERSON, C.M.: Medium chain triglycerides in paediatric practice. Arch. Dis. Childh. $45: 445$ (1970).

6. GRACEY, M., and GOOCH, P.M.: The use of a medium chain triglyceride diet in chyloperitoneum. Aust. paediat. J. $4: 66$ (1968).

7. GRACEY, M., THOMAS, J., SUHARJONO and HENTYANTO HENDARDJI: Assessment of steatorrhoea in malnourished children. J. trop. pediat. and envir. Child Hlth. (in press).

8. GRAHAM, G.G., BAERTH, J.M., CORDANO, A., and MORALES, E. : Lactose-free, medium-chain triglyceride formulas in severe malnutrition. Amer. J. Dis. Child. 126 : 330 (1973). 
9. HOLT, P.R. : Medium chain trigIycerides. A useful adjunct in nutritional therapy. Gastroenterology 53 : 961 (1967).

10. ISSELBACHER, K.J. : Biochemical aspects of fat absorption. Gastroenterology 50 : 78 (1966).

11. SCHEIG, R.: Hepatic matabolism of medium chain fatty acids in Medium Chain Triglycerides' p. 39 edited by J.R. Senior, University of Pennsylvania Press, Philadelphia (1968).
12. SENIOR, J.R. : The place of medium chain triglycerides. Amer. J. med. Sci. 257 : 75 (1969).

13. SUTEDJO, R. : Trends of Paediatric nutrition in developing countries. Paediatr. Indones. 14 : 73 (1974).

14. TAMIR, I., HUNTER, J.0., FOSBROOKE, A.S., and LLOYD, J.K. : Serum and chyle lipids during medium-chain triglyceride feeding in a child with chylothorax. Arch. Dis. Childh. 43 : 302 (1968).

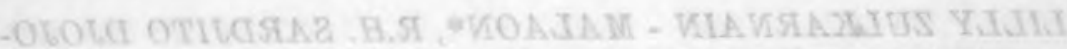

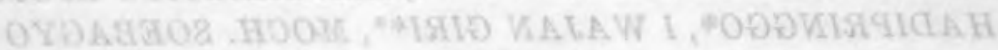 natKIDDKYR}

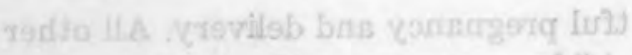
son gd of bergbiamos srisw serblitio holsou (stamoaif right suodguondt Lam

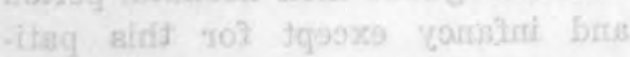

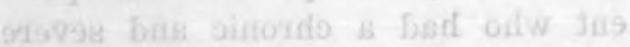

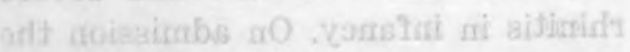

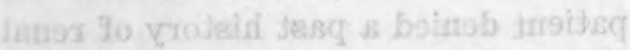

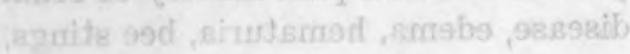

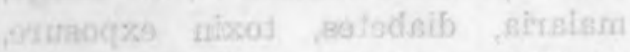

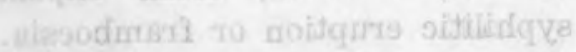

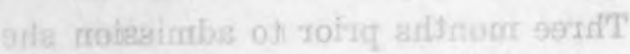

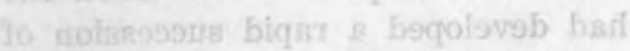

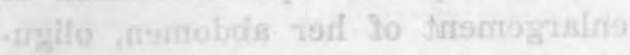

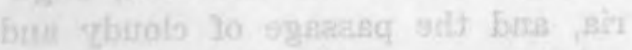

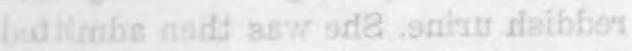
olast anulurt nit intigeorf libms 3 as

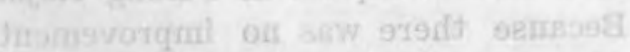

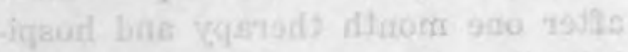

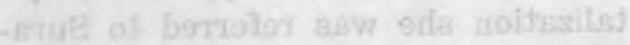

of berobianos apassellis gilti gnom

to agauno gigoloits gldibeou sds sd

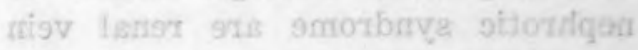
Bifidgre Istinggros bris sieodintondit

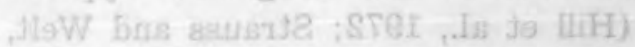

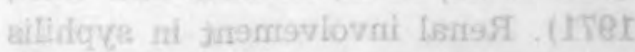
odt tasel Jus rol besingasst meod and

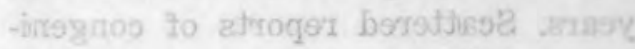

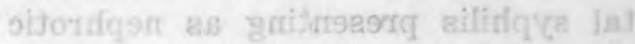

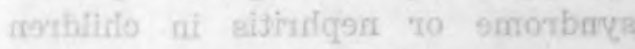

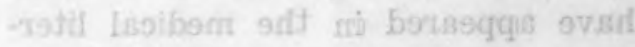

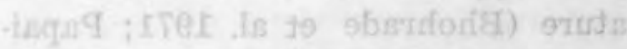
- (roer tha to molinso

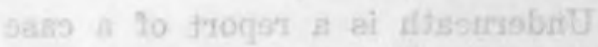

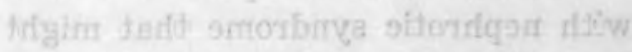
silliniqua lastinegroo of $9 u b$ od Jhocgos sand

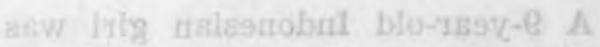
-iqsoll omoteok nd silt of bastimbe -eves ar agdmstqoe no, spadsme, Ind

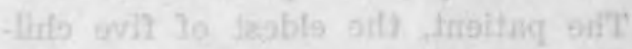

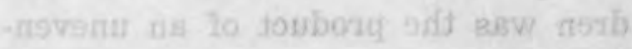
ther vak stst biszisogri 